\title{
Tsafon
}

Revue d'études juives du Nord

$75 \mid 2018$

Exil des langues - Langues d'exil

\section{« La langue allemande restera la langue de mon esprit ». Elias Canetti en Angleterre}

\section{Marion Dufresne}

\section{(2) OpenEdition}

1 Journals

Édition électronique

URL : https://journals.openedition.org/tsafon/580

DOI : $10.4000 /$ tsafon. 580

ISSN : 2609-6420

Éditeur

Association Jean-Marie Delmaire

Édition imprimée

Date de publication : 1 juin 2018

Pagination : 69-80

ISSN : 1149-6630

\section{Référence électronique}

Marion Dufresne, « "La langue allemande restera la langue de mon esprit ». Elias Canetti en

Angleterre », Tsafon [En ligne], 75 | 2018, mis en ligne le 06 mai 2019, consulté le 24 juin 2021. URL

http://journals.openedition.org/tsafon/580 ; DOI : https://doi.org/10.4000/tsafon.580

Tsafon. Revues d'études juives du Nord 


\section{« La langue allemande restera la langue de mon esprit »}

\section{Elias Canetti en Angleterre}

\section{Marion Dufresne*}

Prix Nobel de littérature en 1981, Elias Canetti est né en 1905 à Roustchouk en Bulgarie. Fils aîné d'une famille de Juifs sépharades espagnols, il grandit dans un milieu multiculturel et plurilingue : il note dans son autobiographie qu'il pouvait entendre parler sept ou huit langues différentes dans la journée. Les membres de sa famille parlaient l'espagnol qui était pratiquement le même que celui que parlaient leurs ancêtres des siècles auparavant, quand ils avaient été chassés de la péninsule ibérique.

En 1911, ses parents quittent la Bulgarie pour s'installer en Angleterre, à Manchester où vivait déjà un de ses oncles. Elias est scolarisé la même année et apprend donc facilement l'anglais, sa deuxième langue. Mais à peine deux ans plus tard, en 1913, son père meurt à 31 ans d'une crise cardiaque, laissant une veuve et trois enfants. Mathilde Canetti décide alors de quitter l'Angleterre et de s'installer avec ses fils à Vienne. Après des années d'études passées d'abord en Suisse puis en Allemagne, Elias revient dans la capitale autrichienne en 1925. Il y rencontre Karl Kraus dont l'influence sur ses premières œuvres fut considérable : son unique roman Die Blendung (Auto-da-fé) et ses deux

\footnotetext{
*Université de Lille, SHS.
} 
pièces de théâtre Hochzeit (Noce) et Komödie der Eitelkeit (Comédie des vanités) avec leurs personnages ancrés dans la réalité viennoise, en témoignent. C'est en assistant aux lectures publiques de Kraus que Canetti fait la connaissance de sa première femme, Veza Calderon, qu'il épouse juste avant de fuir la persécution nationale-socialiste en novembre 1938. Le couple s'exile en Angleterre et l'auteur ne retourne vivre sur le continent qu'en 1974 tout en gardant son appartement à Londres et en oscillant entre Zurich et la capitale britannique. Canetti lui-même ne s'est jamais considéré comme un écrivain exilé et souligne qu'il bénéficiait d'un statut particulier parmi les immigrés côtoyés à Londres ${ }^{1}$. Contrairement à ces derniers, il parlait déjà anglais avant son arrivée en 1938 et n'avait aucun effort à fournir pour acquérir la maîtrise d'une nouvelle langue. Compte tenu de cet avantage considérable, on lui a souvent demandé pourquoi il n'avait jamais écrit en anglais, mais toujours rédigé ses œuvres en allemand. N'aurait-il pas été plus facile de les publier dans la langue du pays d'accueil ? La réponse de Canetti a toujours été la même : la question ne s'est jamais posée, à aucun moment écrire en anglais n'a été une option pour lui. Quelles sont les raisons de cet attachement viscéral à l'allemand ?

Devant la réaction quelque peu étonnée de ses interlocuteurs, l'auteur a de nombreuses fois détaillé le rapport spécifique, lié à son histoire personnelle, qu'il entretient avec l'allemand ${ }^{2}$. La langue qu'il parlait pendant son enfance à Roustchouk était l'espagnol ancien des Juifs sépharades, le "ladino ». Mais à côté de cette langue de tous les jours et du bulgare (qu'il ne maîtrisait pas, mais qu'il entendait parler par les jeunes filles employées comme domestiques à la maison), le petit Elias avait également l'occasion d'entendre presque quotidiennement l'allemand. En effet, ses parents, issus de familles de commerçants prospères, étaient passionnés de théâtre (le père d'Elias avait longtemps rêvé de devenir acteur) et leur amour était né de leur intérêt commun pour les représentations du Burgtheater à Vienne où ils avaient fait leurs études. La capitale autrichienne exerçait un attrait considérable sur ces familles aisées qui y envoyaient leurs enfants pour qu'ils apprennent la langue et la culture de l'Empire austro-hongrois. Nostalgiques de

\footnotetext{
${ }^{1}$ Gespräch mit Manfred Durzak, « Akustische Maske und Maskensprung. Materialien zu einer Theorie des Dramas » dans Elias Canetti, Aufsätze, Reden, Gespräche, München, Wien, Carl Hanser Verlag, 2005, p. 298-317.

2 Ibid., Gespräch mit Friedrich Witz, p. 190-220 ; Gespräch mit Rudolf Hartung, p. 228240 ; Gespräch mit Rupprecht Slavko Baur, p. 266-276.
} 
l'atmosphère viennoise et de ce qu'ils avaient vécu ensemble, Mathilde et Jacques Canetti avaient gardé l'habitude de se parler allemand, notamment chaque fois qu'ils voulaient empêcher leurs enfants de les comprendre. Dès que ses parents commençaient à discuter en allemand, Elias se sentait exclu et désemparé ; dans le premier tome de son récit autobiographique, il avoue la jalousie que l'attitude de ses parents fait naître et la rancœur envers sa mère ${ }^{3}$. Mais il mentionne également que les mots allemands défiaient sa curiosité et qu'il souhaitait ardemment arracher son secret à cette langue "magique». Dans La langue sauvée comme dans les entretiens cités, il décrit ses premières tentatives pour parvenir à ses fins : à l'abri des regards et après avoir pris soin de s'enfermer seul dans une pièce, il répète des mots isolés qu'il a retenus, voire des phrases entières en allemand sans en saisir le sens. Dès le départ, la maîtrise de la langue allemande est liée à l'idée d'effort, elle lui résiste, il ne la conquiert qu'au prix de souffrances et d'obstination. Le récit que Canetti fait à maintes reprises de la «méthode» pour le moins singulière et traumatisante qu'emploie, quelques années plus tard, sa mère pour lui apprendre l'allemand confirme cette impression.

La mort subite et prématurée de Jacques Canetti incite sa veuve à quitter Manchester où la famille s'était installée en 1911. Mathilde Canetti veut retourner à Vienne avec ses trois garçons et Elias doit y fréquenter l'école. Comme sa mère ne supporte pas l'idée de le voir redoubler une classe, faute d'une maîtrise satisfaisante de la langue, elle décide de la lui inculquer elle-même et ce en un temps record. Contre toute vraisemblance, son pari fou réussit et, dans son autobiographie, l'écrivain qualifie l'allemand de «langue maternelle acquise sur le tard au prix de véritables souffrances $»{ }^{4}$. Et dans l'interview accordée à Rupprecht Slavko Baur, il affirme : «L'allemand est donc ma langue maternelle au sens premier du terme $»^{5}$. Il comprend également que le mariage de ses parents était issu de l'allemand, c'était la langue de leur amour et ils avaient eux aussi dû lutter pour concrétiser leur union longtemps tenue secrète.

L'idée de devoir mériter une langue et de l'aimer précisément parce qu'elle ne représente pas la voie de la facilité, semble plaire à

\footnotetext{
${ }^{3}$ Elias Canetti, Histoire d'une jeunesse. La langue sauvée, traduit de l'allemand par Bernard Kreiss, Paris, Albin Michel, 1978 (1 $1^{\text {ère }}$ édition), cité dans l'édition de la Pochothèque, Paris, Albin Michel, 2005, p. 29-30.

${ }^{4}$ Ibid., p. 83.

${ }^{5}$ E. Canetti, Aufsätze, Reden, Gespräche, op. cit., p. 268 (traduction MD).
} 
Canetti. C'est ainsi qu'il peut répondre à la question de Rudolf Hartung qui lui demande s'il n'a jamais songé à abandonner la langue allemande et à écrire en anglais : «Non, jamais - pas un seul instant! Écrire en anglais aurait été très facile pour moi, mais je crois que c'est justement la raison pour laquelle cela ne $m$ 'a absolument pas tenté ${ }^{6}$. Langue secrète convoitée, langue magique qui lui semble la plus belle du monde, langue de l'amour de ses parents, l'allemand constitue aussi le fondement de la relation mère-fils. Si elle était auparavant distante et plutôt froide, elle sera désormais on ne peut plus étroite, exclusive et passionnelle. Savoir converser en allemand permet à l'enfant non seulement d'atténuer la douleur de sa mère, mais surtout d'accéder à un statut et à un monde qui lui avaient été jusqu'alors refusés. Elias a l'ambition de remplacer son père et de devenir un interlocuteur à la hauteur des attentes démesurées de sa mère. À l'âge de onze ans, il passe ses soirées à lire et à discuter avec elle des pièces de Shakespeare ou de Schiller. Il dira plus tard que ce sont avant tout ces conversations qui l'ont façonné, qu'il les considère comme la «substance spirituelle que l'on ingère au cours de ses jeunes années, à laquelle on se réfère ensuite et dont on ne peut plus se départir, $[\ldots] »^{7}$.

C'est à cette époque que Canetti, par amour pour sa mère, prend la décision de devenir poète, un poète de langue allemande bien entendu. Exilé vingt-cinq ans plus tard en Angleterre, il se trouve donc dans une situation bien particulière en ce qui concerne ses rapports intimes avec les deux langues qu'il maîtrise le mieux et les liens qui l'attachent à l'allemand priment sur toutes les considérations d'ordre pratique. Canetti a conscience que refuser de s'exprimer en anglais ne pouvait que réduire davantage encore ses chances déjà minces de voir ses œuvres publiées. En dépit de ses difficultés financières, il a fini par consentir à la traduction de son roman Auto-da-fé par Veronica Wedgewood, à la seule condition de pouvoir superviser étroitement le travail de son amie. Lors de la cérémonie de la remise du prix de l'Académie bavaroise des BeauxArts, il déclara en 1969 :

Je ne suis guère qu'un hôte de la langue allemande, que j'ai apprise à huit ans seulement; et que vous m'y déclariez aujourd'hui le bienvenu a pour moi plus de portée que si j'étais né en son champ. Je ne peux même pas prétendre à mériter de m'y être tenu lorsque, il y a plus de trente ans, j'arrivai en Angleterre

\footnotetext{
${ }^{6}$ Ibid., p. 228 (traduction MD).

${ }^{7}$ E. Canetti, La langue sauvée, op. cit., p. 104.
} 
et que je décidai d'y rester. Car pour moi, continuer en Angleterre à écrire en allemand allait de soi, comme respirer et marcher. ${ }^{8}$

Néanmoins il tirait une grande fierté de son obstination à ne pas sacrifier sa langue maternelle. Pour ceux qui, pour des raisons pratiques ou parce qu'ils recherchaient la reconnaissance, avaient consenti à changer de langue, il éprouve avant tout du mépris :

Ils vivaient alors, pour ainsi dire, tout à la vanité de leur nouvel effort, qui ne pouvait avoir de sens que s'il réussissait. Combien de fois n'ai-je pas entendu des gens, doués ou non, s'écrier avec un orgueil presque puéril : "J'écris maintenant en anglais ! »

Aux yeux de Canetti, ils avaient cessé de se battre et avaient capitulé. On touche là un aspect essentiel de l'attitude adoptée par l'auteur: il aurait vécu comme une défaite un abandon de la langue allemande. Dans son allocution lors de la remise du prix Nelly Sachs en 1975, Canetti, après avoir expliqué de nouveau les raisons de son attachement personnel à cette langue, souligne le caractère universel des liens tissés entre les hommes et leur langue :

Il y a beaucoup de choses que les hommes ne veulent tout simplement pas céder et qu'ils sont prêts à défendre avec force et ruse. L'époque des guerres des religions nous a prouvé l'opiniâtreté avec laquelle les gens sont attachés à leur foi, même si elle ne leur apporte que du malheur. Mais je crois que la dernière chose dont un homme ne peut accepter l'interdiction, c'est sa langue. ${ }^{10}$

Le départ du continent avait néanmoins sonné pour Canetti le glas de toute production purement littéraire. On pourrait être tenté d'en chercher la raison dans une réflexion de l'auteur qui propose une analyse très lucide de ce qui advient à la langue en exil et que nous nous permettons de citer presque dans son intégralité :

Il faut à une langue la sûreté d'une longue habitude pour qu'elle ose s'inventer des noms. Le poète en exil, et plus particulièrement le dramaturge, en est affecté et s'en trouve affaibli de différentes façons. Loin de l'atmosphère de sa langue, il lui manque la nourriture familière des noms. [...] Pour l'émigré, par contre, même s'il n'a pas perdu le souvenir de ses noms, il n'y a plus le moindre vent

\footnotetext{
${ }^{8}$ Elias Canetti, La conscience des mots, traduit de l'allemand par Roger Lewinter, Paris, Albin Michel, 1984, p. 197.

${ }^{9}$ Ibid., p. 198.

${ }^{10}$ E. Canetti, Aufsätze, Reden, Gespräche, op. cit., p. 92 (traduction MD).
} 
vivant qui les lui apporte. Il les conserve en lui comme un trésor inanimé, et plus se prolonge son bannissement loin de l'atmosphère qui fut la sienne, plus les doigts sous lesquels se glissent les mots anciens deviennent avares. Il ne reste donc au poète exilé, s'il ne veut pas renoncer à tout ce qu'il est, qu'une issue unique : respirer l'air nouveau jusqu'à ce qu'il lui fasse signe à son tour. Mais cet air-là s'y refuse longtemps ; il esquive une première avance, puis redevient muet. Le poète le ressent et il en est blessé. Il se peut qu'il se bouche les oreilles et que plus aucun nom ne puisse avoir accès à lui. Il se trouve alors de plus en plus dépaysé, et quand il se réveille, c'est toujours le même vieux tas desséché qu'il trouve à côté de lui, et alors il calme sa faim avec les céréales datant de sa jeunesse. ${ }^{11}$

Ces paroles désabusées rappellent la plainte de nombreux auteurs exilés, comme Lion Feuchtwanger ${ }^{12}$ ou Erich Fried, et sonnent comme un écho à la résignation d'Adorno qui, dans Minima Moralia, dépeint l'écrivain exilé comme un homme vaincu, dépossédé de sa langue et sans aucune possibilité d'agir :

Tout intellectuel en émigration est mutilé, aucun n'y échappe, et il fera bien de s'en rendre compte de lui-même s'il ne veut pas en faire cruellement l'expérience, jusque derrière les portes pourtant bien fermées de l'estime qu'il se porte à soi-même. [...] Sa langue est confisquée, et asséchée la dimension historique où s'alimente sa réflexion. ${ }^{13}$

Mais la raison qu'avance Canetti pour expliquer l'abandon du genre fictionnel est d'ordre éthique : ce sont les horreurs de la guerre qui décident l'auteur à s'imposer le silence en tant que romancier et dramaturge pour se consacrer exclusivement à Masse und Macht (Masse et Puissance), œuvre dont il affirmera fièrement qu'elle lui avait permis de «prendre ce siècle à la gorge »" ${ }^{14}$. Cette étude sociologique, fruit de l'ascèse qu'il s'était imposée, livre son analyse du national-socialisme et constitue sa contribution à la lutte contre le fascisme. Rétrospectivement, l'auteur se dit même convaincu que le travail acharné et solitaire auquel

\footnotetext{
${ }^{11}$ Elias Canetti, Le territoire de l'homme, traduit de l'allemand par Armel Guerne, Paris, Albin Michel, 1978 ( $1^{\text {ère }}$ édition), cité dans l'édition de la Pochothèque, Paris, Albin Michel, 2005, p. 1013-1014.

${ }^{12}$ Lors d'une interview à la radio française en 1938, Feuchtwanger déclare : «C'est un malheur pour un écrivain d'être émigré parce qu'il est séparé du fleuve vivant de sa langue maternelle ».

${ }^{13}$ Theodor Adorno, Minima Moralia. Réflexions sur la vie mutilée, traduit de l'allemand par Eliane Kaufholz et Jean-René Ladmiral, Paris, Payot \& Rivages, collection Petite Bibliothèque Payot, 2001, p. 37.

${ }^{14}$ E. Canetti, Le territoire de l'homme, op. cit., p. 1197.
} 
il s'adonnait en exil, n'aurait jamais été possible sans le recours à la langue allemande. Depuis 1928, Canetti travaillait à la rédaction de ce livre. Il avait évidemment formulé en allemand ses observations et ses analyses des nombreux ouvrages consultés en vue de ce projet. Toutes les conversations, tous les échanges qu'il avait pu avoir à son propos avaient eu lieu en cette langue. Les artistes et les intellectuels qu'il fréquentait à Vienne, Robert Musil, Hermann Broch, Fritz Wotruba, Alban Berg et surtout Abraham Sonne parlaient allemand. Ne plus pratiquer l'allemand lui aurait semblé comme une rupture impardonnable avec ceux qui avaient suivi son travail et dont l'opinion lui importait beaucoup. Maintenir ce dialogue vivant en dépit de l'éloignement géographique, mais aussi envers les circonstances et les difficultés quotidiennes était un puissant moteur et explique certainement en partie la persévérance de Canetti.

Dans l'entretien accordé à Rudolf Hartung en 1971, Canetti souligne en outre les avantages qui découlent selon lui de la coexistence de deux langues, réservée chacune à des domaines très distincts et ne se mélangeant pas. L'anglais était la langue de tous les jours, celle dont se servaient les journalistes pour rédiger leurs articles et les gens en général pour gérer leurs affaires quotidiennes. Pratiquer l'anglais ne demandait aucun effort, alors qu'écrire en allemand permettait une réflexion aiguë. L'exil aurait alors plutôt favorisé l'élaboration de l'œuvre théorique et rendu aux mots allemands toute leur vigueur ${ }^{15}$. Le travail de réflexion s'était ainsi doublé de l'effort continu de maintenir vivante la langue interdite. C'est cette symbiose qui a permis d'assurer une continuité dans la rupture. Pour Canetti, il s'agit d'un véritable acte de résistance et de révolte :

C'était comme si tous les jours on s'arrogeait avec aplomb le droit d'enfreindre l'interdiction que l'on ne saurait accepter. C'est en réitérant sans relâche cette démarche que l'on refusait de valider les nouvelles reçues du monde extérieur. Chaque mot, même s'il prenait sa source à la douleur et au désespoir, se transformait en espoir en se fixant sur le papier. ${ }^{16}$

Il est intéressant de remarquer qu'en 1960, juste après avoir enfin terminé la rédaction de Masse und Macht, Canetti semble avoir douté de son choix et regrette que son « esprit n'ait jamais adopté l'habit anglais ». Délivré du poids de son ouvrage auquel il a consacré plus de trente ans

${ }^{15}$ E. Canetti, Aufsätze, Reden, Gespräche, op. cit., p. 313.
${ }^{16}$ Ibib., p. 93 (traduction MD). 
de son existence, l'auteur a gagné son combat et éprouve le désir de «commencer une vie dans une autre langue ${ }^{17}$. C'était une envie passagère, Canetti est resté un poète de langue allemande.

"La langue allemande restera la langue de mon esprit », note-t-il en 1944 et il n'a eu de cesse de le répéter également à ceux qui, à l'instar de Manfred Durzak, l'interrogeaient sur les effets néfastes de l'exil sur la langue exilée. Durzak compare la pièce de théâtre Die Befristeten (Les sursitaires), créée en Angleterre et publiée en 1954, aux deux ouvrages précédents de l'auteur, le drame Hochzeit (Noce) et la comédie Komödie der Eitelkeit (La comédie des vanités), tous deux nés sur le sol autrichien et publiés respectivement en 1935 et 1937. Ces œuvres ancrées dans la tradition viennoise, représentée notamment par Karl Kraus que Canetti vénérait et par Nestroy, mettent en scène des personnages se distinguant nettement les uns des autres par un langage qui leur est propre et qui les caractérise mieux que toute autre description. Canetti parle à ce sujet de « masque acoustique » et en fait un procédé dramatique de choix. Est-ce que son abandon serait, comme le suggère Durzak, une conséquence de la situation d'exilé qui, en 1954, est toujours celle de l'auteur ${ }^{18}$ ?

Le drame Les Sursitaires est en effet une pièce qui n'accorde plus aucune importance au fait que la façon de s'exprimer devrait refléter le niveau intellectuel, l'origine sociale ou encore le caractère des personnages. Canetti a recours à une langue très neutre, partagée par tous les acteurs. Lors de cette interview, Canetti souligne la singularité de cette pièce qu'il a écrite alors qu'une personne aimée se mourait lentement ${ }^{19}$. Il insiste sur l'intentionnalité de son choix et sur sa volonté de s'exprimer dans une langue «quasi blanche", «sans aucune coloration $»^{20}$. Elle seule lui semble en adéquation avec la mort, donnée universelle au centre de la pièce. En revanche, il est important de ne pas la confondre avec la langue épurée de Masse et Puissance à laquelle il tendait bien avant d'avoir quitté le vieux continent. Quelles sont alors les stratégies déployées pour ne jamais interrompre ce dialogue intime avec la langue qui seule est jugée digne et apte à véhiculer ce qui représente pour l'auteur le cheminement et l'aboutissement de sa pensée ?

\footnotetext{
${ }^{17}$ Elias Canetti, Notes de Hampstead 1954-1971, traduit de l'allemand par Walter Weideli, Paris, Albin Michel, 1997, p. 30.

${ }^{18}$ E. Canetti, Aufsätze, Reden, Gespräche, op. cit., p. 313.

${ }^{19}$ Cette personne était Frieda Benedikt, plus connue sous le pseudonyme d'Anna Sebastian, auteure de plusieurs romans en anglais et longtemps une des maîtresses de Canetti.

${ }^{20}$ E. Canetti, Aufsätze, Reden, Gespräche, op. cit., p. 313.
} 
Certes, Elias Canetti a emporté en exil son impressionnante bibliothèque qui compte de nombreux ouvrages rédigés en allemand. Mais il découvre rapidement que même si les œuvres des poètes restent les représentants de la langue, ce sont les mots eux-mêmes, dans leur isolement et dans leur singularité, qui s'imposent avec une vigueur nouvelle. Dans son allocution à l'Académie des Beaux-Arts, l'auteur avoue une activité dont il avait honte à l'époque et qu'il s'efforçait de cacher à son entourage. Il cédait régulièrement à une envie irrésistible de noircir des pages entières de mots allemands qui n'avaient pas de lien entre eux. Il ajoute qu'il se sentait particulièrement heureux lors de cette occupation inhabituelle. Dans une de ses notes, il avait déjà confessé se sentir vraiment Zuhause [chez soi] lorsqu'il notait des mots allemands alors qu'autour de lui, tout le monde parlait anglais ${ }^{21}$. Ces «crises de mots », comme il désigne ces assauts soudains, sont pour Canetti le signe d'une pression devenue trop forte sur la langue. Face à l'omniprésence de la langue du pays d'accueil, la langue de l'homme en exil se comporte comme un être vivant qui se sentirait délaissé, voire même renié. La « crise de mots » serait alors une sorte de vengeance, mais également un rappel à l'ordre de celui qui néglige la langue menacée ${ }^{22}$. Ce que Canetti décrit pour la langue allemande en Angleterre rappelle ce que les philosophes Deleuze et Guattari établissent comme les caractéristiques d'une littérature mineure. L'allemand se trouve affecté d'un «fort coefficient de déterritorialisation ${ }^{23}$ et l'auteur cherche à la protéger contre l'absorption par la langue majeure et dominante. En fixant les mots sur le papier, Canetti cherche à les préserver contre des déformations en tous genres afin de les garder intacts dans la dynamique qui leur est propre.

Continuer à écrire en allemand était pour l'homme Elias Canetti un moyen de préserver non seulement la langue allemande de l'oubli, mais, faisant corps avec elle, de sauver également sa vie. Sa passion pour l'allemand était née de sa connaissance du seul mot dont ses parents aient consenti à lui délivrer la signification et qui fut Wien [Vienne]. Il est chargé du poids de l'amour que se portaient les parents et qui est un lien que Canetti établit entre la langue allemande et le sentiment amoureux (n'oublions pas que c'est à Vienne que Canetti rencontre Veza et Karl

\footnotetext{
${ }^{21}$ E. Canetti, Le territoire de l'homme, op. cit., p. 1196.

${ }^{22}$ E. Canetti, La conscience des mots, op. cit., p. 197-201.

${ }^{23}$ Gilles Deleuze et Félix Guatarri, Kafka. Pour une littérature mineure, Paris, Minuit, 1975, p. 29.
} 
Kraus). Quant à la langue anglaise, bien que l'écrivain la juge froide et souvent méprisante ${ }^{24}$, elle était celle du pays où son père avait été heureux. C'est en Angleterre et en anglais qu'Elias apprend à lire et que, dans les discussions au sujet des livres que lui apporte son père, naît son goût pour les histoires et les mythes. Indestructible est « la base morale » que son père a ainsi jetée ${ }^{25}$. Canetti se devait de la concilier avec les fondements de ce qu'il appelle sa « sphère privée » qui, dans son cas, se confond avec l'allemand: «[...] la sphère privée, mais que je n'ai aucunement aménagée avec confort, où je voudrais que tout se déroule scrupuleusement et de façon responsable, est la langue allemande $»^{26}$. C'est certainement une des raisons pour lesquelles l'auteur s'est aussi durablement installé en Angleterre et c'est également ce qui perce dans cette note rédigée à l'intention de son amie, la peintre Marie-Louise von Motesiczky, en 1942 : « Il aurait aimé être son propre père et en même temps sa propre mère $\gg{ }^{27}$.

Lutter pour sauvegarder son intégrité était aussi sa façon de résister à l'effondrement général qu'il pouvait observer autour de lui dans un pays en guerre. Que cette résistance prenne corps dans des mots allemands, rétablit la langue exilée dans ses droits. $\mathrm{Ne}$ jamais l'abandonner allait finir par revêtir l'importance d'une mission plus universelle.

À l'instar de ses ancêtres, Juifs sépharades chassés de l'Espagne par le décret d'Alhambra en 1492 et qui avaient emporté avec eux leur langue, Canetti a sauvegardé la langue du peuple juif expulsé d'Allemagne. Comme il l'explique à Horst Bienek, l'auteur attachait une importance capitale à cette filiation :

Je n'aurais pas supporté que quelqu'un - et surtout pas Hitler - m'impose le choix de la langue dans laquelle j'écris. [...] À trente-trois ans, j'ai dû quitter Vienne et j'emportais avec moi l'allemand comme ils avaient fait à l'époque avec l'espagnol. Il se peut que je sois le seul écrivain qui a un lien aussi étroit

\footnotetext{
${ }^{24}$ Voir à ce sujet: Marion Dufresne, « Elias Canetti et l'image de l'Angleterre », dans André Combes, Anne-Marie Corbin et Irina Fougeron (sous la dir. de), Images de l'altérité, Lille, CeGes, coll. UL3 travaux et recherches, 2002, p. 105-117.

${ }^{25}$ Elias Canetti, Les années anglaises, traduit de l'allemand par Bernard Kreiss, Paris, Albin Michel, 2005, p. 224-225.

${ }^{26}$ E. Canetti, La conscience des mots, op. cit., p. 201.

${ }^{27}$ Elias Canetti, Aufzeichnungen für Marie-Louise, Frankfurt a. M., S. Fischer Verlag, 2009, p. 57 (traduction MD).
} 
avec les langues de ces deux grandes expulsions. Il ne faut pas perturber une configuration aussi curieuse. ${ }^{28}$

Une remarque faite dans une lettre que Canetti adressa à son frère Georges en 1945 met déjà l'accent sur le rôle de la langue dans la quête identitaire de l'écrivain. Elias aimerait écrire à Georges dans le vieil espagnol de sa famille; il lui confie même nourrir l'ambition d'écrire un jour en espagnol, mais seulement une fois l'anglais parfaitement maîtrisé $^{29}$. On comprend donc aisément que le choix de la langue allemande était aussi celui de la langue qui lui obéissait le mieux, la seule qui lui permettait d'exprimer ses pensées avec la clarté et la précision recherchées.

"La langue allemande restera la langue de mon esprit », affirme Canetti, mais ce n'est que la première partie de sa confession. «La langue allemande restera la langue de mon esprit, et cela parce que je suis juif $\gg{ }^{30}$, écrit-il. Il affirme vouloir garder en lui ce qui reste de l'Allemagne détruite. En sa qualité de Juif, il apporte un héritage à l'humanité entière. Alors que l'Allemagne est dévastée et ses habitants à la recherche de la langue qu'on leur a volée, Canetti veut reconnaître sa dette et restituer au peuple allemand la langue qu'il a gardée pure :

Mais il y a que maintenant il doit leur langue aux Allemands. Il l'a maintenue saine, mais il faut maintenant qu'il la lâche et la rende avec amour et gratitude, avec les intérêts et les intérêts composés. ${ }^{31}$

L'écrivain établit même un étonnant parallèle entre les Juifs et les Allemands lorsqu'en 1945 ces derniers doivent s'avouer vaincus :

Lorsque viendra le printemps, le deuil des Allemands sera un puits intarissable, qui ne leur permettra plus de se distinguer des Juifs. Hitler a fait des Allemands, en très peu d'années, des Juifs; et le mot «Allemand» est devenu à présent aussi douloureux que celui de « Juif ${ }^{32}$

Qu'il soit possible de combattre l'effroyable réalité que les nationaux-socialistes avaient voulu imposer, voilà l'espoir qui n'a jamais

\footnotetext{
${ }^{28}$ E. Canetti, Aufsätze, Reden, Gespräche, op. cit., p. 173 (traduction MD).

${ }^{29}$ Karen Lauer, Kristian Wachinger (éd.), Veza \& Elias Canetti, Briefe an Georges, München, Wien, Hanser Verlag, 2006, p. 164.

${ }^{30}$ E. Canetti, Le territoire de l'homme, op. cit., p. 1032.

${ }^{31}$ Ibid., p. 1049.

${ }^{32}$ Ibid., p. 1038.
} 
quitté Canetti. Il le puisait dans la langue allemande. En tant que poète, Canetti défend vigoureusement sa place dans la lignée des grands écrivains de langue allemande. Cette revendication peut étonner: à l'époque, Canetti est un inconnu en Angleterre et ignoré dans les pays germanophones. Mais il est certain que cette reconnaissance lui est due, convaincu qu'un jour on saura gré aux Allemands d'avoir prêté leur langue au Juif Elias Canetti.

Lui, un de ces Juifs qui ne devraient plus être là, un paria que l'on avait voulu exterminer, se proclame le gardien de la langue allemande et ce précisément en sa qualité de Juif. C'est en accomplissant ce devoir que l'auteur reste fidèle à la « base morale » posée par son père et s'avère digne du nom de poète. Il apporte ainsi sa pierre à cette lutte acharnée qu'il a livrée sa vie durant à son pire ennemi : la mort. Sauver la langue allemande était sauver la vie. Si Canetti a eu la chance d'échapper aux camps d'extermination et aux fours crématoires, il avait une dette envers tous ceux et toutes celles qui y avaient péri. Canetti n'était pas un Juif pratiquant, mais en 1944 il avoue lutter contre « la plus grande tentation spirituelle de ma vie, la seule contre laquelle il m'est très dur de lutter, c'est d'être juif totalement »; ce sont ces millions de morts qui semblent le supplier de les rejoindre, et « qui aurait le cœur de leur dire non ? ${ }^{33}$ Mais ces morts, le poète les déplore parmi tous les peuples et de tous les côtés. La mort est le fléau, l'ennemi qui frappe sans distinction. Canetti qui était l'ennemi déclaré de la mort, devait trouver une réponse à cette question qu'il se posait en 1944 :

Ai-je le droit de me fermer aux Russes sous prétexte qu'il existe des Juifs, aux Chinois parce qu'ils sont loin de nous, aux Allemands parce qu'ils sont possédés du diable ? Ne puis-je pas continuer à être à eux tous, comme auparavant, tout en étant Juif quand même ? ${ }^{34}$

Laisser une œuvre qui serait encore lue dans cent ans était le vœu qu'il avouait sans fausse modestie. C'était sa façon de témoigner de son époque et de s'opposer à la barbarie.

\footnotetext{
${ }^{33}$ Ibid., p. 1030.

${ }^{34}$ Ibid.
} 